\title{
NUMERICAL AND HYDRAULIC EXPERIMENTS ON BORE PRESSURE DUE TO TSUNAMI
}

\author{
Tatsuto Kimura, Tokyo Electric Power Service Co., Ltd., kimura tatsu@tepsco.co.jp \\ Masahiro Masuko, Tokyo Electric Power Service Co., Ltd., emasu@tepsco.co.jp \\ Naoki Fujii, Tokyo Electric Power Service Co., Ltd., dfujii@tepsco.co.jp \\ Hideki Kaida, Central Research Institute of Electric Power Industry, h-kaida@criepi.denken.or.jp \\ Naoto Kihara, Central Research Institute of Electric Power Industry, kihara@criepi.denken.or.jp
}

\section{INTRODUCTION}

The 2011 Tohoku earthquake tsunami struck a wide area of the northeastern coast of Japan, and many coastal structures and buildings were damaged by the tsunami. Most of the buildings were damaged by the tsunami wave pressure. After the tsunami, characteristics of tsunami waive pressures have been investigated by many researcher, and are being clarified. As shown in previous studies, there are three regimes charactering the vertical pressure profiles. The first one is the impulsive pressure, which is observed just after the tsunami-bore impacted structures. In this regime, strong hydrodynamic pressures are generated by the fluid-solid impact process. After that, the bore pressure is observed, and both the hydrodynamic and hydrostatic pressures contribute the pressure profile. After that, the flow near the structures reaches a quasi-steady state, and the pressure profile becomes hydrostatic. Most of the evaluation equations of tsunami wave pressure proposed by the previous studies can be used against the impulsive pressures and the pressures in the quasisteady-state regime. On the other hand, the characteristics and quantitative evaluations of the bore pressure remain immature. In this study, in order to clarify the characteristics of the bore pressure, experiments on the bore pressure are carried out, and furthermore, three-dimensional numerical simulations are also carried out.

\section{EXPERIMENTS}

The experiments were carried out by using the LargeScale Tsunami Physical Simulator of CRIEPI. A vertical wall with $1.5 \mathrm{~m}$ height was set on a flat bed in the test section. A gate was installed in the upstream side of the test section. The upstream area of the gate was a storage tank. A bore was generated by rapidly opening the gate, which attacked the wall and streamed through the side of the wall. The experiment was carried out by varying the initial water depth in the storage tank in five ways. The vertical distributions of pressure were measured on the center line of the upstream surface of the wall. The pressures were measured at 23 heights between 0.01 and $1.49 \mathrm{~m}$ from the bed. Velocities and water depths were also measured at some points in the test section. In addition to the experiments, experiments in the absence of the wall were carried out, and the velocities and water depths were measured.

\section{CHARACTERISTICS OF BORE PRESSURE}

Shortly after the incident bore impacted the wall, a splash jet was generated. Then, the jet fell onto the incident bore, and a turbulent bore similar to a hydraulic jump was generated and traveled toward the upstream side. A snapshot of the upstream-traveling turbulent bore and the vertical distribution of the pressure of the upstream surface of the wall at the same time are shown in Figure 1. The pressure profile is a complicated form. With increasing distance from the bed, the pressure decreases $(z<0.15 \mathrm{~m})$ and then increases slightly $(0.15 \mathrm{~m}<z<0.3 \mathrm{~m})$. Then, the pressure decreases to a negative value $(0.3 \mathrm{~m}<z<0.9 \mathrm{~m})$. After that, the pressure approaches zero $(0.9 \mathrm{~m}<z<1.1 \mathrm{~m})$. In another study by the authors, they tried to express the pressure profiles by using a theory of the turbulent bore.

\section{NUMERICAL EXPERIMENTS}

In order to reproduce the aforementioned bore pressure profile, we carried out numerical experiments using the three-dimensional fluid dynamics code OpenFOAM. The interFoam solver, which is a model of incompressible two-phase flow, was adopted. In the numerical experiments, two types of wall-boundary condition (slip/nonslip) and two types of the turbulent model (k- $\varepsilon$ model/Smagorinsky model) were used. By selecting the nonslip boundary condition, the characteristic pressure profiles near the bed, where pressure decreases once and then increases, can be reproduced (Figure 2). However, the negative pressure, which was observed in the hydraulic experiment, could not be reproduced in any cases carried out in this study.

(a)
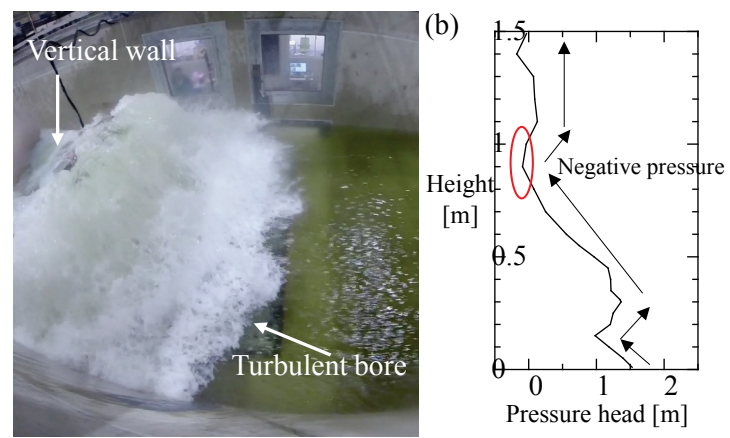

Figure 1 - A snapshot of the upstream traveling turbulent bore (a), and the vertical distribution of the pressure of the upstream surface of the wall at the time (b).
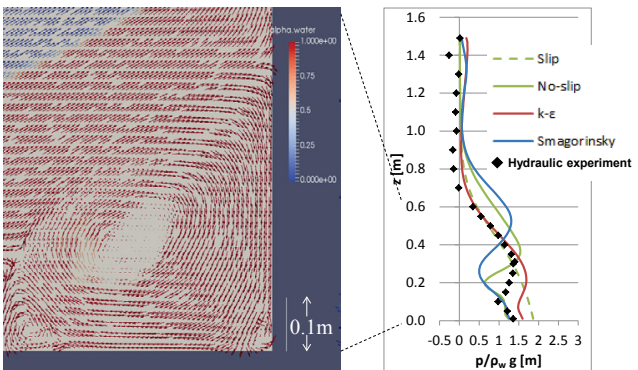

Figure 2 - A snapshot of the velocity vectors in front of the wall predicted by the case with the Smagorinsky model (a), and comparison of the vertical distribution of between predicted and measured pressure (b). 